\title{
RULED MINIMAL LAGRANGIAN SUBMANIFOLDS OF COMPLEX PROJECTIVE 3-SPACE*
}

\author{
J. BOLTON ${ }^{\dagger}$ AND L. VRANCKEN ${ }^{\ddagger}$
}

\begin{abstract}
We show how a ruled minimal Lagrangian submanifold of complex projective 3-space may be used to construct two related minimal surfaces in the 5 -sphere.
\end{abstract}

Key words. Complex projective space, Lagrangian submanifold, sphere, minimal surface

AMS subject classifications. 53B25, 53B20

1. Introduction. In previous papers [1], [2] we showed how a Lagrangian submanifold $M$ of complex projective 3 -space $\mathbb{C} P^{3}(4)$ satisfying Chen's equality [7] but having no totally geodesic points may be used to construct a minimal surface in the unit 5 -sphere $S^{5}(1)$ with ellipse of curvature a circle.

In this paper, we replace the assumption concerning Chen's equality with the assumption that $M$ is minimal and admits a foliation by asymptotic curves, that is to say curves with vanishing normal curvature. In fact, these curves turn out to be geodesics of $\mathbb{C} P^{3}(4)$ (hence our description of $M$ as a ruled submanifold of $\mathbb{C} P^{3}(4)$ ), and we show that the local construction referred to above may be applied to $M$ to give two minimal surfaces in $S^{5}(1)$ whose ellipses of curvature are not circles. We also show that these minimal surfaces are related by a transform which generalises that of the polar (see [3], [9]) for linearly full minimal surfaces in $S^{5}(1)$ whose ellipses of curvature are circles. In a forthcoming paper [4], we will show that this transform may be defined for all non totally geodesic minimal surfaces in $S^{5}(1)$.

2. Ruled minimal Lagrangian submanifolds. Let $M$ be a Lagrangian submanifold of $\mathbb{C} P^{3}(4)$. That is to say, if $J$ is the complex structure of $\mathbb{C} P^{3}(4)$, then $J$ maps the tangent bundle of $M$ onto the normal bundle. Let $\tilde{\nabla}$ denote the Riemannian connection on $\mathbb{C} P^{3}(4)$, and $\nabla, \nabla^{\perp}$ the induced connections on $M$ and the normal bundle of $M$. Let $h(X, Y)=\tilde{\nabla}_{X} Y-\nabla_{X} Y$ denote the second fundamental form of $M$, and, if $N$ is a normal vector field, let $A_{N}(X)=-\tilde{\nabla}_{X} N+\nabla_{X}^{\perp} N$ denote the corresponding shape operator. If $\langle$,$\rangle denotes the Fubini-Study metric on \mathbb{C} P^{3}(4)$, then $[5,8]$, the cubic form

$$
C(X, Y, Z)=\langle h(X, Y), J Z\rangle=\left\langle A_{J Z}(X), Y\right\rangle
$$

is symmetric in $X, Y$ and $Z$. In particular,

$$
A_{J X}(Y)=A_{J Y}(X)=-J h(X, Y) .
$$

We now assume that $M$ admits a smooth unit length vector field $\mathbf{e}_{1}$ whose integral curves are asymptotic curves in $M$, that is to say they have zero normal curvature, so that

$$
h\left(\mathbf{e}_{1}, \mathbf{e}_{1}\right)=0 .
$$

\footnotetext{
*Received October 22, 2003; accepted for publication March 22, 2004. The authors are grateful to the London Mathematical Society for financial support during the preparation of this paper.

${ }^{\dagger}$ Department of Mathematical Sciences, University of Durham, Durham DH1 3LE, U.K. (john.bolton@durham.ac.uk).

${ }_{\ddagger}^{\ddagger}$ LAMATH, ISTV2, Université de Valenciennes, Campus du Mont Houy, 59313 Valenciennes Cedex 9, France (luc.vrancken@univ-valenciennes.fr).
} 
If $A_{J \mathbf{e}_{1}}$ vanishes identically at some point $p \in M$, then $M$ satisfies Chen's equality at $p$ (see [7]), and the situation in which this holds on an open subset of $M$ has been discussed in [1] and [2]. Since we are dealing with a local theory here, we will from now on assume that $M$ does not satisfy Chen's equality at any point.

It follows from (2) and (3) that $A_{J \mathbf{e}_{1}} \mathbf{e}_{1}=0$, so we may choose eigenvectors $\mathbf{e}_{2}$ and $\mathbf{e}_{3}$ of $A_{J \mathbf{e}_{1}}$ such that $\left\{\mathbf{e}_{1}, \mathbf{e}_{2}, \mathbf{e}_{3}\right\}$ is an orthonormal basis of the tangent space of $M$. Let $\lambda_{2}, \lambda_{3}$ be the eigenvalues corresponding to $\mathbf{e}_{2}, \mathbf{e}_{3}$ respectively.

We now assume that $M$ is minimal, so that

$$
0=\left\langle h\left(\mathbf{e}_{2}, \mathbf{e}_{2}\right)+h\left(\mathbf{e}_{3}, \mathbf{e}_{3}\right), J \mathbf{e}_{1}\right\rangle=\left\langle A_{J \mathbf{e}_{1}} \mathbf{e}_{2}, \mathbf{e}_{2}\right\rangle+\left\langle A_{J \mathbf{e}_{1}} \mathbf{e}_{3}, \mathbf{e}_{3}\right\rangle=\lambda_{2}+\lambda_{3} .
$$

Thus $\lambda_{2}=-\lambda_{3}=\lambda$, where we may assume that $\lambda$ is a strictly positive function on $M$ and $\mathbf{e}_{2}, \mathbf{e}_{3}$ are smooth unit vector fields.

If we put $a=\left\langle A_{J \mathbf{e}_{2}} \mathbf{e}_{2}, \mathbf{e}_{2}\right\rangle, b=\left\langle A_{J \mathbf{e}_{2}} \mathbf{e}_{2}, \mathbf{e}_{3}\right\rangle$ then it is easy to check using (2), (3) and (4) that, with respect to the orthonormal basis $\left\{\mathbf{e}_{1}, \mathbf{e}_{2}, \mathbf{e}_{3}\right\}$, we have the following matrix expressions.

$$
\begin{gathered}
A_{J \mathbf{e}_{1}}=\left(\begin{array}{ccc}
0 & 0 & 0 \\
0 & \lambda & 0 \\
0 & 0 & -\lambda
\end{array}\right), \\
A_{J \mathbf{e}_{2}}=\left(\begin{array}{ccc}
0 & \lambda & 0 \\
\lambda & a & b \\
0 & b & -a
\end{array}\right), \\
A_{J \mathbf{e}_{3}}=\left(\begin{array}{ccc}
0 & 0 & -\lambda \\
0 & b & -a \\
-\lambda & -a & -b
\end{array}\right) .
\end{gathered}
$$

Let $z_{j}^{i}$ be the connection 1 -forms on $M$ defined by

$$
\nabla \mathbf{e}_{j}=z_{j}^{i} \mathbf{e}_{i}
$$

and define the connection coefficients $z_{k j}^{i}$ by

$$
z_{j}^{i}\left(\mathbf{e}_{k}\right)=z_{k j}^{i}
$$

so that

$$
z_{k j}^{i}=\left\langle\nabla_{\mathbf{e}_{k}} \mathbf{e}_{j}, \mathbf{e}_{i}\right\rangle=-z_{k i}^{j} .
$$

We use the fundamental equations of submanifold theory, namely the Gauss, Codazzi and Ricci equations, to find relations between $z_{j k}^{i}, a, b$ and $\lambda$. However, for Lagrangian submanifolds, the Gauss and Ricci equations are equivalent.

First consider the Codazzi equations, namely,

$\nabla_{X}^{\perp}(h(Y, Z))-h\left(\nabla_{X} Y, Z\right)-h\left(Y, \nabla_{X} Z\right)=\nabla_{Y}^{\perp}(h(X, Z))-h\left(\nabla_{Y} X, Z\right)-h\left(X, \nabla_{Y} Z\right)$.

If we apply $J$ to this expression and take (2) into account, we see that that the Codazzi equations are equivalent to

$$
\nabla_{X}\left(A_{J Y} Z\right)-A_{J\left(\nabla_{X} Y\right)} Z-A_{J Y}\left(\nabla_{X} Z\right)=\nabla_{Y}\left(A_{J X} Z\right)-A_{J\left(\nabla_{Y} X\right)} Z-A_{J X}\left(\nabla_{Y} Z\right) .
$$


Equations (5), (6) and (7) may be used to show that (11) is equivalent to the following system (12)-(19).

$$
\begin{gathered}
z_{11}^{2}=z_{11}^{3}=0, \quad z_{12}^{3}=z_{21}^{3}=-z_{31}^{2}, \quad z_{21}^{2}=z_{31}^{3} \\
\mathbf{e}_{1}(\lambda)=-2 z_{21}^{2} \lambda \\
\mathbf{e}_{2}(\lambda)=-2 z_{32}^{3} \lambda \\
\mathbf{e}_{3}(\lambda)=2 z_{22}^{3} \lambda \\
\mathbf{e}_{1}(a)=2 b z_{12}^{3}-a z_{21}^{2}-2 z_{32}^{3} \lambda \\
\mathbf{e}_{1}(b)=-2 a z_{12}^{3}-b z_{21}^{2}+2 z_{22}^{3} \lambda \\
\mathbf{e}_{3}(a)-\mathbf{e}_{2}(b)=3 a z_{22}^{3}+3 b z_{32}^{3}+4 z_{12}^{3} \lambda \\
\mathbf{e}_{3}(b)+\mathbf{e}_{2}(a)=3 b z_{22}^{3}-3 a z_{32}^{3}-2 z_{21}^{2} \lambda
\end{gathered}
$$

In particular, we note from (12) that $\nabla_{\mathbf{e}_{1}} \mathbf{e}_{1}=0$, so that, by (3), the integral curves of $\mathbf{e}_{1}$ are geodesics in $C P^{3}(4)$. We have thus proved the following lemma.

Lemma 1. Let $M$ be a minimal Lagrangian submanifold of $\mathbb{C} P^{3}(4)$. If $M$ admits a foliation by asymptotic curves then these curves are geodesics of $\mathbb{C} P^{3}(4)$, so that $M$ is a ruled submanifold.

We next investigate the Gauss curvature equation, which states that the curvature tensor $R$ of $\nabla$ is given by

$$
R(X, Y) Z=\langle Y, Z\rangle X-\langle X, Z\rangle Y+\left(A_{h(Y, Z)} X-A_{h(X, Z)} Y\right) .
$$

Taking (2) into account, the above equation is equivalent to

$$
R(X, Y) Z=\langle Y, Z\rangle X-\langle X, Z\rangle Y+\left[A_{J X}, A_{J Y}\right] Z .
$$

Using (5), (6), (7) and (12), we find that (20) is equivalent to the following system (21)-(24).

$$
\begin{gathered}
\mathbf{e}_{1}\left(z_{12}^{3}\right)=-2 z_{12}^{3} z_{21}^{2}, \quad \mathbf{e}_{1}\left(z_{21}^{2}\right)=-1+\left(z_{12}^{3}\right)^{2}-\left(z_{21}^{2}\right)^{2}+\lambda^{2}, \\
\mathbf{e}_{1}\left(z_{22}^{3}\right)=-\mathbf{e}_{3}\left(z_{21}^{2}\right)-z_{21}^{2} z_{22}^{3}, \quad \mathbf{e}_{1}\left(z_{32}^{3}\right)=\mathbf{e}_{2}\left(z_{21}^{2}\right)-z_{21}^{2} z_{32}^{3}, \\
\mathbf{e}_{2}\left(z_{12}^{3}\right)=-\mathbf{e}_{3}\left(z_{21}^{2}\right)+2 b \lambda, \quad \mathbf{e}_{3}\left(z_{12}^{3}\right)=\mathbf{e}_{2}\left(z_{21}^{2}\right)-2 a \lambda, \\
\mathbf{e}_{2}\left(z_{32}^{3}\right)=\mathbf{e}_{3}\left(z_{22}^{3}\right)+2 a^{2}+2 b^{2}-1-3\left(z_{12}^{3}\right)^{2}-\left(z_{21}^{2}\right)^{2}-\left(z_{22}^{3}\right)^{2}-\left(z_{32}^{3}\right)^{2}+\lambda^{2} .
\end{gathered}
$$


The Gauss, Codazzi and Ricci equations provide a full set of integrability conditions, so we have the following theorem.

THEOREM 1. Let $\left\{\mathbf{e}_{1}, \mathbf{e}_{2}, \mathbf{e}_{3}\right\}$ be an orthonormal moving frame on a simplyconnected Riemannian manifold $M$, and let $\left\{z_{k j}^{i}\right\}$ be the connection coefficients of the corresponding Riemannian connection. If there exist functions $\lambda>0, a, b$ on $M$ satisfying (12)-(19) and (21)-(24) then $M$ may be isometrically immersed as a ruled minimal Lagrangian submanifold of $\mathbb{C} P^{3}(4)$ with shape operator A given by (5), (6) and (7). Moreover the immersion is unique up to holomorphic isometries of $\mathbb{C} P^{3}(4)$.

We now show the existence of such submanifolds $M$ of $\mathbb{C} P^{3}(4)$. In fact, we will show in a forthcoming paper [4] that a solution $f(x, y)$ to the sinh-Gordon equation

$$
f_{x x}+f_{y y}+4 \sinh f=0
$$

determines a ruled minimal Lagrangian submanifold of $\mathbb{C} P^{3}(4)$ with the property that the distribution orthogonal to the rulings is integrable. Indeed, let $f$ be such a function and let $\mu(t, x, y)=\cos t \sinh f+\cosh f$. Then define a Riemannian metric on a suitable open subset of $\mathbb{R}^{3}$ by taking $\mathbf{e}_{1}=-2(\partial / \partial t), \mathbf{e}_{2}=\mu^{-1 / 2}(\partial / \partial x), \mathbf{e}_{3}=\mu^{-1 / 2}(\partial / \partial y)$ to be an orthonormal moving frame. It follows easily from the Koszul formula that the non-zero connection coefficients of the corresponding Riemannian connection are given by

$$
z_{21}^{2}=-z_{22}^{1}=z_{31}^{3}=-z_{33}^{1}=-\frac{\mu_{t}}{\mu}, \quad z_{32}^{3}=-z_{33}^{2}=\frac{\mu_{x}}{2 \mu^{3 / 2}}, \quad z_{22}^{3}=-z_{23}^{2}=-\frac{\mu_{y}}{2 \mu^{3 / 2}},
$$

and, in particular, $\left\{\mathbf{e}_{2}, \mathbf{e}_{3}\right\}$ span an integrable distribution. Then taking

$$
\lambda=1 / \mu, \quad a=\frac{f_{x} \sin t}{2 \mu^{3 / 2}}, \quad b=\frac{f_{y} \sin t}{2 \mu^{3 / 2}},
$$

it may be checked that (12)-(19) and (21)-(24) are all satisfied, so we may apply Theorem 1 to prove the existence of a corresponding ruled minimal Lagrangian submanifold of $\mathbb{C} P^{3}(4)$.

Returning now to the general situation, let $\mathbf{E}_{0}$ be a local horizontal lift of a ruled minimal Lagrangian submanifold $M$ of $\mathbb{C} P^{3}(4)$ to the total space of the Hopf fibration $\pi: S^{7}(1) \rightarrow \mathbb{C} P^{3}(4)$, where $S^{7}(1)$ is the unit sphere in $\mathbb{R}^{8}=\mathbb{C}^{4}$. The existence of such a lift follows from a result of Reckziegel [10], and any two such lifts $\mathbf{E}_{0} \tilde{\mathbf{E}}_{0}$ are related by $\tilde{\mathbf{E}_{0}}=e^{i \theta} \mathbf{E}_{0}$, where $\theta$ is a constant.

For $j=1,2,3$, let $\mathbf{E}_{j}$ be the image under the derivative $d \mathbf{E}_{0}$ of $\mathbf{e}_{j}$, and let $\mathcal{E}=\left(\mathbf{E}_{0}, \mathbf{E}_{1}, \mathbf{E}_{2}, \mathbf{E}_{3}\right)$ be the map from $M$ to the unitary group $U(4)$ so constructed.

We now write down the moving frame equations of $\mathcal{E}$. In fact, if $\omega_{1}, \omega_{2}, \omega_{3}$ is the dual frame to $\mathbf{e}_{1}, \mathbf{e}_{2}, \mathbf{e}_{3}$, a routine calculation using (5)-(9) and (12) shows that

$$
d \mathcal{E}=\mathcal{E}(\alpha+i \beta)
$$

where

$$
\alpha=\left(\begin{array}{cccc}
0 & -\omega_{1} & -\omega_{2} & -\omega_{3} \\
\omega_{1} & 0 & -z_{21}^{2} \omega_{2}+z_{12}^{3} \omega_{3} & -z_{12}^{3} \omega_{2}-z_{21}^{2} \omega_{3} \\
\omega_{2} & z_{21}^{2} \omega_{2}-z_{12}^{3} \omega_{3} & 0 & -z_{12}^{3} \omega_{1}-z_{22}^{3} \omega_{2}-z_{32}^{3} \omega_{3} \\
\omega_{3} & z_{12}^{3} \omega_{2}+z_{21}^{2} \omega_{3} & z_{12}^{3} \omega_{1}+z_{22}^{3} \omega_{2}+z_{32}^{3} \omega_{3} & 0
\end{array}\right)
$$


and

$$
\beta=\left(\begin{array}{cccc}
0 & 0 & 0 & 0 \\
0 & 0 & \lambda \omega_{2} & -\lambda \omega_{3} \\
0 & \lambda \omega_{2} & \lambda \omega_{1}+a \omega_{2}+b \omega_{3} & b \omega_{2}-a \omega_{3} \\
0 & -\lambda \omega_{3} & b \omega_{2}-a \omega_{3} & -\lambda \omega_{1}-a \omega_{2}-b \omega_{3}
\end{array}\right)
$$

Note that taking a different horizontal lift $\mathbf{E}_{0}$ would imply that we multiply $\mathbf{E}_{0}$ (and thus also $\mathbf{E}_{1}, \mathbf{E}_{2}$ and $\mathbf{E}_{3}$ ) by a factor $e^{i \theta}$, where $\theta$ is a constant. Thus we may choose a lift for which $\mathcal{E}$ lies in $S U(4)$ at some point. It then follows from (26) and $(27)$ that $\mathcal{E}$ always lies in $S U(4)$ so, by choosing a suitable horizontal lift $E_{0}$, we may assume that

$$
\mathcal{E}: M \rightarrow S U(4) .
$$

We now compose $\mathcal{E}$ with a suitably chosen standard double-cover of $S O(6)$ by $S U(4)$ to obtain a map $\mathcal{U}: M \rightarrow S O(6)$. In fact, if we let $V$ be the 6 -dimensional real subspace of the second exterior power $\wedge^{2} \mathbb{C}^{4}$ of $\mathbb{C}^{4}$ spanned by

$$
\begin{array}{rr}
\mathbf{U}_{1}=\frac{1}{\sqrt{2}}\left(\mathbf{E}_{0} \wedge \mathbf{E}_{3}+\mathbf{E}_{1} \wedge \mathbf{E}_{2}\right), & \mathbf{U}_{2}=\frac{1}{\sqrt{2}}\left(\mathbf{E}_{0} \wedge \mathbf{E}_{1}+\mathbf{E}_{2} \wedge \mathbf{E}_{3}\right), \\
\mathbf{U}_{3}=\frac{1}{\sqrt{2}}\left(\mathbf{E}_{0} \wedge \mathbf{E}_{2}+\mathbf{E}_{3} \wedge \mathbf{E}_{1}\right), & \mathbf{U}_{4}=\frac{1}{i \sqrt{2}}\left(\mathbf{E}_{0} \wedge \mathbf{E}_{1}-\mathbf{E}_{2} \wedge \mathbf{E}_{3}\right), \\
\mathbf{U}_{5}=\frac{1}{i \sqrt{2}}\left(\mathbf{E}_{0} \wedge \mathbf{E}_{2}-\mathbf{E}_{3} \wedge \mathbf{E}_{1}\right), & \mathbf{U}_{6}=\frac{1}{i \sqrt{2}}\left(\mathbf{E}_{0} \wedge \mathbf{E}_{3}-\mathbf{E}_{1} \wedge \mathbf{E}_{2}\right),
\end{array}
$$

then $V$ is a constant subspace. If we extend the standard inner product on $\mathbb{C}^{4}$ to $\wedge^{2} \mathbb{C}^{4}$ and identify $V$ with $\mathbb{E}^{6}$ by choosing an orthonormal basis of $V$, then we obtain our required map $\mathcal{U}=\left(U_{1}, \ldots, U_{6}\right): M \rightarrow S O(6)$.

We now write down the moving frame equations of $\mathcal{U}$. In fact, if

$$
d \mathcal{U}=\mathcal{U} \Omega
$$

for a $6 \times 6$ matrix $\Omega$ of 1 -forms on $M$, then a calculation using (26) and (27) shows that

$$
\Omega=\left(\begin{array}{ccc}
0 & \left(z_{12}^{3}-1\right) \omega_{2}+z_{21}^{2} \omega_{3} & \left(z_{12}^{3}+1\right) \omega_{1}+z_{22}^{3} \omega_{2}+z_{32}^{3} \omega_{3} \\
\left(1-z_{12}^{3}\right) \omega_{2}-z_{21}^{2} \omega_{3} & 0 & -z_{21}^{2} \omega_{2}+\left(z_{12}^{3}-1\right) \omega_{3} \\
-\left(z_{12}^{3}+1\right) \omega_{1}-z_{22}^{3} \omega_{2}-z_{32}^{3} \omega_{3} & z_{21}^{2} \omega_{2}+\left(1-z_{12}^{3}\right) \omega_{3} & 0 \\
\lambda \omega_{3} & 0 & -\lambda \omega_{2} \\
-b \omega_{2}+a \omega_{3} & -\lambda \omega_{2} & -\lambda \omega_{1}-a \omega_{2}-b \omega_{3} \\
\lambda \omega_{1}+a \omega_{2}+b \omega_{3} & \lambda \omega_{3} & -b \omega_{2}+a \omega_{3} \\
-\lambda \omega_{3} & b \omega_{2}-a \omega_{3} & -\lambda \omega_{1}-a \omega_{2}-b \omega_{3} \\
0 & \lambda \omega_{2} & -\lambda \omega_{3} \\
\lambda \omega_{2} & \lambda \omega_{1}+a \omega_{2}+b \omega_{3} & b \omega_{2}-a \omega_{3} \\
0 & -z_{21}^{2} \omega_{2}+\left(z_{12}^{3}+1\right) \omega_{3} & -\left(z_{12}^{3}+1\right) \omega_{2}-z_{21}^{2} \omega_{3} \\
z_{21}^{2} \omega_{2}-\left(z_{12}^{3}+1\right) \omega_{3} & 0 & \left(1-z_{12}^{3}\right) \omega_{1}-z_{22}^{3} \omega_{2}-z_{32}^{3} \omega_{3}
\end{array}\right)
$$


It is clear from the above that $d \mathbf{U}_{2}\left(\mathbf{e}_{1}\right)=0$, while

$$
\begin{aligned}
& d \mathbf{U}_{2}\left(\mathbf{e}_{2}\right)=\left(-1+z_{12}^{3}\right) \mathbf{U}_{1}+z_{21}^{2} \mathbf{U}_{3}-\lambda \mathbf{U}_{5}, \\
& d \mathbf{U}_{2}\left(\mathbf{e}_{3}\right)=z_{21}^{2} \mathbf{U}_{1}+\left(1-z_{12}^{3}\right) \mathbf{U}_{3}+\lambda \mathbf{U}_{6} .
\end{aligned}
$$

It follows that the image of $\mathbf{U}_{2}$ is a surface $S$ in $S^{5}(1)$, and we now show that this is a minimal surface.

Lemma 2. The vectors $X=d \mathbf{U}_{2}\left(\mathbf{e}_{2}\right)$ and $Y=d \mathbf{U}_{2}\left(\mathbf{e}_{3}\right)$ are perpendicular and have the same (non-zero) length.

TheOREM 2. The image $S$ of $\mathbf{U}_{2}$ is a minimal surface in $S^{5}(1)$.

Proof. Let $I I$ denote the second fundamental form of $S$ in $S^{5}(1)$. It follows from Lemma 2 that we need only check that $I I(X, X)+I I(Y, Y)=0$, or, equivalently, that $d X\left(\mathbf{e}_{2}\right)+d Y\left(\mathbf{e}_{3}\right)$ is a linear combination of $\mathbf{U}_{2}, X$ and $Y$. In fact, a calculation using (14), (15), (23) and (33) shows that the component of $d X\left(\mathbf{e}_{2}\right)+d Y\left(\mathbf{e}_{3}\right)$ perpendicular to $\mathbf{U}_{2}$ is equal to $-z_{32}^{3} X+z_{22}^{3} Y$, from which the result follows. $\square$

We now investigate the ellipse of curvature $E$ of $S$. Recall that the ellipse of curvature at a point $p$ of a minimal surface is that (possibly degenerate) ellipse in the first normal space given by

$$
E=\{I I(Z, Z) \mid Z \text { is a unit tangent vector to } S \text { at } p\} .
$$

Lemma 3. The ellipse of curvature at any point of $S$ is not a circle. The direction of the minor axis is given by $I I(X, X)$ and that of the major axis by $I I(X, Y)$.

Proof. We first note that $2 I I(X, X)$ (resp. $2 I I(X, Y))$ is equal to the component of $d X\left(\mathbf{e}_{2}\right)-d Y\left(\mathbf{e}_{3}\right)$ (resp. $\left.\left.d X\left(\mathbf{e}_{3}\right)\right)+d Y\left(\mathbf{e}_{2}\right)\right)$ perpendicular to $S$. In order to facilitate the calculations, which we carried out using Mathematica, we let

$$
\mathbf{K}_{1}=d X\left(\mathbf{e}_{2}\right)-d Y\left(\mathbf{e}_{3}\right)+3\left(z_{22}^{3} Y+z_{32}^{3} X\right),
$$

and

$$
\mathbf{K}_{2}=d X\left(\mathbf{e}_{3}\right)+d Y\left(\mathbf{e}_{2}\right)+3\left(z_{32}^{3} Y-z_{22}^{3} X\right),
$$

so that $2 I I(X, X)$ and $2 I I(X, Y)$ are the components of $\mathbf{K}_{1}$ and $\mathbf{K}_{2}$ perpendicular to $S$. A calculation shows that

$$
\begin{aligned}
& \mathbf{K}_{1}=\mu_{2} \mathbf{U}_{1}+\mu_{1} \mathbf{U}_{3}+\mu_{3} \mathbf{U}_{5}+\mu_{4} \mathbf{U}_{6}, \\
& \mathbf{K}_{2}=\mu_{1} \mathbf{U}_{1}-\mu_{2} \mathbf{U}_{3}-4 \lambda \mathbf{U}_{4}+\mu_{4} \mathbf{U}_{5}-\mu_{3} \mathbf{U}_{6},
\end{aligned}
$$

where

$$
\begin{aligned}
& \mu_{1}=4\left(z_{22}^{3}-z_{12}^{3} z_{22}^{3}+z_{21}^{2} z_{32}^{3}\right)+\mathbf{e}_{3}\left(z_{12}^{3}\right)+\mathbf{e}_{2}\left(z_{21}^{2}\right), \\
& \mu_{2}=4\left(z_{21}^{2} z_{22}^{3}-z_{32}^{3}+z_{12}^{3} z_{32}^{3}\right)+\mathbf{e}_{2}\left(z_{12}^{3}\right)-\mathbf{e}_{3}\left(z_{21}^{2}\right), \\
& \mu_{3}=2\left(b\left(1-z_{12}^{3}\right)-a z_{21}^{2}\right), \\
& \mu_{4}=2\left(a\left(z_{12}^{3}-1\right)-b z_{21}^{2}\right) .
\end{aligned}
$$


It is clear from (36) and (37) that $\mathbf{K}_{1}$ and $\mathbf{K}_{2}$ are orthogonal vectors, and from (34) and (35) that

$$
\left(\mathbf{K}_{1}, X\right)=\left(\mathbf{K}_{2}, Y\right) \quad \text { and } \quad\left(\mathbf{K}_{1}, Y\right)=-\left(\mathbf{K}_{2}, X\right),
$$

where ( , ) denotes the standard inner product on $\mathbb{R}^{6}$. Hence the components of $\mathbf{K}_{1}$ and $\mathbf{K}_{2}$ tangential to $S$ are orthogonal and have the same length. It now follows that $I I(X, X)$ and $I I(X, Y)$ are also orthogonal, so that $I I(X, X)$ and $I I(X, Y)$ lie along the axes of the ellipse of curvature. Also, it is clear from (36) and (37) that $\left|\mathbf{K}_{2}\right|^{2}-\left|\mathbf{K}_{1}\right|^{2}=16 \lambda^{2}$, implying that

$$
|I I(X, Y)|^{2}-|I I(X, X)|^{2}=4 \lambda^{2} .
$$

Hence the ellipse of curvature is not a circle since $\lambda \neq 0$.

We note from (42) that there is a positive function $\phi$ such that

$$
|I I(X, Y)|=2 \lambda \cosh \phi \quad \text { and } \quad|I I(X, X)|=2 \lambda \sinh \phi .
$$

We may express the eccentricity $e$ of the ellipse of curvature in terms of $\phi$. In fact,

$$
\begin{aligned}
e & =\sqrt{1-\frac{|I I(X, X)|^{2}}{|I I(X, Y)|^{2}}} \\
& =\operatorname{sech} \phi .
\end{aligned}
$$

We have seen that $X$ and $Y$ determine geometrically significant directions on $S$, so we would therefore expect that $d X\left(\mathbf{e}_{1}\right)$ and $d Y\left(\mathbf{e}_{1}\right)$ are scalar multiples of $X$ and $Y$ respectively. In fact, it follows from (21), (13) and (33) that $d X\left(\mathbf{e}_{1}\right)=-z_{21}^{2} X$ and $d Y\left(\mathbf{e}_{1}\right)=-z_{21}^{2} Y$.

We now determine conditions on $M$ in order that $S$ lies in a totally geodesic $S^{3}(1)$ in $S^{5}(1)$.

Theorem 3. Let $S$ be the minimal surface in $S^{5}(1)$ determined by $\mathbf{U}_{2}$. Then the following conditions are equivalent.

- (i) The surface $S$ is contained in a totally geodesic $S^{3}(1)$ in $S^{5}(1)$.

- (ii) The ellipse of curvature of $S$ is degenerate at each point of $S$.

- (iii) The vector field $\mathbf{e}_{1}$ on $M$ is a Killing vector field.

- (iv) $\left\langle\nabla_{\mathbf{e}_{2}} \mathbf{e}_{1}, \mathbf{e}_{2}\right\rangle=0$.

- (v) $\left\langle\nabla_{\mathbf{e}_{3}} \mathbf{e}_{1}, \mathbf{e}_{3}\right\rangle=0$.

Remark. Minimal Lagrangian submanifolds $M$ admitting a unit length Killing vector field whose integral curves are geodesics in $\mathbb{C} P^{3}(4)$ are investigated in [6]. In particular, explicit examples of minimal Lagrangian tori admitting such a vector field are constructed.

Proof. The equivalence of $(i v)$ and $(v)$ is immediate from (12).

Minimality of $S$, together with the Codazzi equations for $S$ show that $(i)$ holds if and only if $I I(X, X) \equiv 0$ on an open subset of $S$ or, equivalently, (ii) holds.

On the other hand, (iii) holds if and only if $\mathbf{e}_{1}$ satisfies the Killing equations, namely $\left\langle\nabla_{U} \mathbf{e}_{1}, V\right\rangle+\left\langle\nabla_{V} \mathbf{e}_{1}, U\right\rangle=0$ for all vectors $U, V$ tangential to $M$. It follows from (12) that this holds if and only if (iv) holds.

Hence, we may prove the theorem by showing that the vector $\mathbf{K}_{1}$ given by (36) is a linear combination of $X$ and $Y$ if and only if $z_{21}^{2}=0$. 
We first assume that $z_{21}^{2}=0$. In this case, using (23) we see that

$\mathbf{K}_{1}=2\left(2 z_{32}^{3}\left(z_{12}^{3}-1\right)+b \lambda\right) \mathbf{U}_{1}-2\left(2 z_{22}^{3}\left(z_{12}^{3}-1\right)+a \lambda\right) \mathbf{U}_{3}+2 b\left(1-z_{12}^{3}\right) \mathbf{U}_{5}+2 a\left(z_{12}^{3}-1\right) \mathbf{U}_{6}$.

This is a linear combination of $X$ and $Y$ if and only if both the following equations hold.

$$
a\left(z_{12}^{3}-1\right)^{2}=2 z_{22}^{3} \lambda\left(z_{12}^{3}-1\right)+a \lambda^{2}
$$

and

$$
b\left(z_{12}^{3}-1\right)^{2}=2 z_{32}^{3} \lambda\left(z_{12}^{3}-1\right)+b \lambda^{2} .
$$

Using (21), these equations simplify to

$$
a z_{12}^{3}=\lambda z_{22}^{3}
$$

and

$$
b z_{12}^{3}=\lambda z_{32}^{3} .
$$

However, it follows from (12) that $\left[\mathbf{e}_{1}, \mathbf{e}_{2}\right]=0$, and, applying this to $z_{12}^{3}$ using (13), (17), (21) and (23), we obtain (46). Similarly, $\left[\mathbf{e}_{1}, \mathbf{e}_{3}\right]=0$, and, applying this to $z_{12}^{3}$, we obtain (47). Thus $\mathbf{K}_{1}$ is a linear combination of $X$ and $Y$ as required.

Conversely, assume that $\mathbf{K}_{1}$ is a linear combination of $X$ and $Y$. It then follows from (34), (35) and (36) that

$$
\lambda \mu_{1}=-\mu_{3} z_{21}^{2}+\mu_{4}\left(1-z_{12}^{3}\right),
$$

and

$$
\lambda \mu_{2}=\mu_{3}\left(1-z_{12}^{3}\right)+\mu_{4} z_{21}^{2} .
$$

We may use the above two equations, together with (23) to obtain the following algebraic expressions for $\mathbf{e}_{2}\left(z_{21}^{2}\right), \mathbf{e}_{3}\left(z_{21}^{2}\right), \mathbf{e}_{2}\left(z_{12}^{3}\right)$ and $\mathbf{e}_{3}\left(z_{12}^{3}\right)$.

$$
\begin{aligned}
& \lambda \mathbf{e}_{2}\left(z_{21}^{2}\right)=-\left(z_{12}^{3}-1\right)^{2} a+2\left(z_{12}^{3}-1\right)\left(b z_{21}^{2}+\lambda z_{22}^{3}\right)+a\left(\left(z_{21}^{2}\right)^{2}+\lambda^{2}\right)-2 \lambda z_{21}^{2} z_{32}^{3}, \\
& \lambda \mathbf{e}_{3}\left(z_{21}^{2}\right)=-\left(z_{12}^{3}-1\right)^{2} b+2\left(z_{12}^{3}-1\right)\left(-a z_{21}^{2}+\lambda z_{32}^{3}\right)+b\left(\left(z_{21}^{2}\right)^{2}+\lambda^{2}\right)+2 \lambda z_{21}^{2} z_{22}^{3}, \\
& \lambda \mathbf{e}_{2}\left(z_{12}^{3}\right)=\left(z_{12}^{3}-1\right)^{2} b+2\left(z_{12}^{3}-1\right)\left(a z_{21}^{2}-\lambda z_{32}^{3}\right)+b\left(-\left(z_{21}^{2}\right)^{2}+\lambda^{2}\right)-2 \lambda z_{21}^{2} z_{22}^{3}, \\
& \lambda \mathbf{e}_{3}\left(z_{12}^{3}\right)=-\left(z_{12}^{3}-1\right)^{2} a+2\left(z_{12}^{3}-1\right)\left(b z_{21}^{2}+\lambda z_{22}^{3}\right)+a\left(\left(z_{21}^{2}\right)^{2}-\lambda^{2}\right)-2 \lambda z_{21}^{2} z_{32}^{3} .
\end{aligned}
$$

We now consider the integrability conditions for $\lambda$. In fact, the only one we will need is obtained by applying $\nabla_{\mathbf{e}_{2}} \mathbf{e}_{3}-\nabla_{\mathbf{e}_{3}} \mathbf{e}_{2}-\left[\mathbf{e}_{2}, \mathbf{e}_{3}\right]$ to $\lambda$ and equating the answer to zero. Carrying out this process using (12)-(15), we obtain

$$
\mathbf{e}_{2}\left(z_{22}^{3}\right)+\mathbf{e}_{3}\left(z_{32}^{3}\right)=2 z_{12}^{3} z_{21}^{2} .
$$

Using the above equations and (12)-(19), (21)-(24), a calculation (for which we used Mathematica) shows that the integrability condition obtained by applying $\nabla_{\mathbf{e}_{2}} \mathbf{e}_{3}-\nabla_{\mathbf{e}_{3}} \mathbf{e}_{2}-\left[\mathbf{e}_{2}, \mathbf{e}_{3}\right]$ to $z_{12}^{3}$ and equating the answer to zero reduces to $z_{21}^{2}=0$. This completes the proof of the theorem. 
We now return to the general situation governed by the ruled minimal Lagrangian submanifold $M$ of $\mathbb{C} P^{3}(4)$. We note that the arguments applied to $\mathbf{U}_{2}$ may be used to show that the image $\hat{S}$ of $\mathbf{U}_{4}$ is also a minimal surface in $S^{5}(1)$. We now investigate the relation between the two minimal surfaces $S$ and $\hat{S}$.

LEMma 4. If $S$ is contained in a totally geodesic $S^{3}(1)$ then $\hat{S}$ is the polar of $S$ in the sense of Lawson [9].

Proof. In this situation, $I I(X, X)=0$, so that $\mathbf{K}_{1}$ is a linear combination of $X$ and $Y$. We also have from Theorem 3 that $z_{21}^{2}=0$, so it follows from (34), (35), (36) and (37) that $2 I I(X, Y)$, the component of $\mathbf{K}_{2}$ perpendicular to $X$ and $Y$, is equal to $-4 \lambda \mathbf{U}_{4}$. In particular, $\hat{S}$ is in the totally geodesic $S^{3}(1)$ containing $S$ and at each point is orthogonal to $S$ and the tangent space to $S$. Thus $\hat{S}$ is the polar of $S$.

We now assume that $S$ is not contained in a totally geodesic $S^{3}(1)$. Theorem 3 , together with real analyticity of minimal surfaces imply that, by restricting to an open dense subset of $M$, we may assume that $z_{21}^{2}$ is a nowhere vanishing function. Therefore, by replacing $\mathbf{e}_{1}$ with $-\mathbf{e}_{1}$ (and interchanging $\mathbf{e}_{2}$ and $\mathbf{e}_{3}$ in order to keep $\lambda$ positive) if necessary, we may also assume that $z_{21}^{2}$ is a strictly positive function. We now let $\mathbf{N}$ be the unit vector in $\mathbb{R}^{6}$ such that

$\left\{\mathbf{U}_{2}, X, Y, I I(X, X), I I(X, Y), \mathbf{N}\right\}$ is a positively oriented orthogonal frame of $\mathbb{R}^{6}$.

It follows from (34), (35) and (36) that $\mathbf{U}_{4}$ is orthogonal to $\mathbf{U}_{2}, X, Y$, and $I I(X, X)$, and so is a linear combination of $I I(X, Y)$ and $\mathbf{N}$. Also, it follows from (37) that $\left(\mathbf{U}_{4}, I I(X, Y)\right)=-2 \lambda$ so that, using the positive function $\phi$ introduced in (43), we see that

$$
\mathbf{U}_{4}=-\operatorname{sech} \phi \frac{I I(X, Y)}{|I I(X, Y)|}+\epsilon \tanh \phi \mathbf{N}
$$

where $\epsilon= \pm 1$.

In order to determine the sign of $\epsilon$, we compute the determinant of $\left(\mathbf{U}_{2}, X, Y, I I(X, X), I I(X, Y), \mathbf{U}_{4}\right)$. In fact,

$$
\begin{aligned}
4 \operatorname{det}\left(\mathbf{U}_{2}, X, Y, I I(X, X), I I(X, Y), \mathbf{U}_{4}\right)=\operatorname{det}\left(\mathbf{U}_{2}, X, Y, \mathbf{K}_{1}, \mathbf{K}_{2}, \mathbf{U}_{4}\right) \\
=-\left(\mu_{1}{ }^{2}+\mu_{2}{ }^{2}\right) \lambda^{2}+2\left(\left(z_{12}^{3}-1\right)\left(\mu_{1} \mu_{4}+\mu_{2} \mu_{3}\right)+z_{21}^{2}\left(\mu_{1} \mu_{3}-\mu_{2} \mu_{4}\right)\right) \lambda \\
-\left(\mu_{3}{ }^{2}+\mu_{4}{ }^{2}\right)\left(\left(z_{12}^{3}-1\right)^{2}+\left(z_{21}^{2}\right)^{2}\right) .
\end{aligned}
$$
by

Regarding this as a quadratic equation in $\lambda$, we see that its discriminant is given

$$
-\left(\left(\mu_{4} \mu_{2}+\mu_{3} \mu_{1}\right)\left(z_{12}^{3}-1\right)+z_{21}^{2}\left(\mu_{2} \mu_{3}-\mu_{1} \mu_{4}\right)\right)^{2} .
$$

This is always non-positive, implying that

$$
\operatorname{det}\left(\mathbf{U}_{2}, X, Y, I I(X, X), I I(X, Y), \mathbf{U}_{4}\right) \leq 0,
$$

from which it follows that $\epsilon=-1$.

We remark that the minimal surface $\hat{S}$ determined by $\mathbf{U}_{4}$ may be determined directly from $S$ together with a choice of direction along the major axis of the ellipse of curvature $E$ of $S$. In fact, the unit vector $\mathbf{N}$ determined by (55) does not depend on the choice of basis $\{X, Y\}$ of the tangent space of $S$, so if $V$ is the unit vector in 
the direction determined by $I I(X, Y)$ along the major axis of $E$ then we may define ^: $S \rightarrow \hat{S}$, where

$$
\hat{p}=(-\operatorname{sech} \phi V-\tanh \phi \mathbf{N})(p), \quad p \in S .
$$

We will call this the (-)transform of $S$. This, together with a related construction called the (+)transform, may be described geometrically as follows. Let $E$ be the ellipse of curvature of $S$ at a point $p \in S$ and let $P$ be the 3-plane orthogonal to $\mathbf{U}_{2}$ and its tangent space. Let $R_{\theta}$ be the rotation of $P$ about the minor axis of $E$ through an angle $\theta, 0 \leq \theta \leq \pi / 2$, such that $R_{\theta}(V)$ makes an acute angle with $\mathbf{N}$ and having the property that the orthogonal projection of $R_{\theta}(E)$ onto the plane containing $E$ is a circle. Then the inverse rotation $R_{-\theta}$ has a similar geometric effect on $E$, and we define the (+)transform and (-)transform of $S$ by setting

$$
p^{+}=R_{\theta}(\mathbf{N})=(-\operatorname{sech} \phi V+\tanh \phi \mathbf{N})(p), \quad p \in S,
$$

and

$$
p^{-}=R_{-\theta}(-\mathbf{N})=(-\operatorname{sech} \phi V-\tanh \phi \mathbf{N})(p), \quad p \in S .
$$

Thus $\mathbf{U}_{4}$ is obtained by applying the (-)transform to the minimal surface determined by $\mathbf{U}_{2}$, and we now show that $\mathbf{U}_{2}$ is obtained by applying the (+)transform to the minimal surface determined by $\mathbf{U}_{4}$. We begin by noting that if, in our construction, we replace $\mathbf{e}_{3}$ by $-\mathbf{e}_{3}$ then a suitable lift to $S U(4)$ gives the map $\tilde{\mathcal{U}}=\left(\tilde{\mathbf{U}}_{1}, \ldots, \tilde{\mathbf{U}}_{6}\right): M \rightarrow S O(6)$ where

$$
\tilde{\mathbf{U}}_{1}=\mathbf{U}_{6}, \quad \tilde{\mathbf{U}}_{2}=-\mathbf{U}_{4}, \quad \tilde{\mathbf{U}}_{3}=-\mathbf{U}_{5}, \quad \tilde{\mathbf{U}}_{4}=\mathbf{U}_{2}, \quad \tilde{\mathbf{U}}_{5}=\mathbf{U}_{3}, \quad \tilde{\mathbf{U}}_{6}=-\mathbf{U}_{1} .
$$

Thus, from Theorem $3, \hat{S}$ is not contained in a totally geodesic $S^{3}(1)$. Also,

$$
\tilde{\mathbf{U}}_{4}=-\operatorname{sech} \tilde{\phi} \frac{\tilde{I I}(\tilde{X}, \tilde{Y})}{|\tilde{I}(\tilde{X}, \tilde{Y})|}-\tanh \tilde{\phi} \tilde{\mathbf{N}}
$$

where $\tilde{\phi}>0$ is such that sech $\tilde{\phi}$ is the eccentricity of the ellipse of curvature of the surface $\tilde{S}$ determined by $\tilde{\mathbf{U}}_{2}, \tilde{X}=d \tilde{\mathbf{U}}_{2}\left(\mathbf{e}_{2}\right), \tilde{Y}=d \tilde{\mathbf{U}}_{2}\left(-\mathbf{e}_{3}\right), \tilde{I I}$ is the second fundamental form of $\tilde{S}$, and $\tilde{\mathbf{N}}$ is the unit vector in $\mathbb{R}^{6}$ such that $\left\{\tilde{\mathbf{U}}_{2}, \tilde{X}, \tilde{Y}, \tilde{I} I(\tilde{X}, \tilde{X}), \tilde{I} I(\tilde{X}, \tilde{Y}), \tilde{\mathbf{N}}\right\}$ is a positively oriented orthogonal frame of $\mathbb{R}^{6}$.

Now let $\hat{X}=d \mathbf{U}_{4}\left(\mathbf{e}_{2}\right), \hat{Y}=d \mathbf{U}_{4}\left(\mathbf{e}_{3}\right)$, and let $\hat{I I}$ be the second fundamental form of $\hat{S}$. Then $\hat{I} I(\hat{X}, \hat{X})=-\tilde{I} I(\tilde{X}, \tilde{X})$ is along the minor axis of the ellipse of curvature of $\hat{S}$, while $\hat{I}(\hat{X}, \hat{Y})=\tilde{I} I(\tilde{X}, \tilde{Y})$ is along the major axis. Now let $\hat{\mathbf{N}}$ be the unit vector in $\mathbb{R}^{6}$ such that $\left\{\mathbf{U}_{4}, \hat{X}, \hat{Y}, \hat{I I}(\hat{X}, \hat{X}), \hat{I I}(\hat{X}, \hat{Y}), \hat{\mathbf{N}}\right\}$ is a positively oriented orthogonal basis of $\mathbb{R}^{6}$. It then follows from (58) that $\tilde{\mathbf{N}}=-\hat{\mathbf{N}}$, and from (58) and (59) that if $\hat{\phi}>0$ is such that sech $\hat{\phi}$ is the eccentricity of the ellipse of curvature of $\hat{S}$, then

$$
\mathbf{U}_{2}=-\operatorname{sech} \hat{\phi} \frac{\hat{I} I(\hat{X}, \hat{Y})}{|\hat{I} I(\hat{X}, \hat{Y})|}+\tanh \hat{\phi} \hat{\mathbf{N}}
$$

Thus, taking the direction along the major axis of the ellipse of curvature of $\hat{S}$ to be that determined by $\hat{I} I(\hat{X}, \hat{Y})$, then applying the $(+)$ transform to $\hat{S}$ gives us $S$.

The following theorem summarises the results of the paper.

Theorem 4. A ruled minimal Lagrangian submanifold $M$ of $\mathbb{C} P^{3}(4)$ defines two minimal surfaces $S$ and $\hat{S}$ in $S^{5}(1)$. These surfaces are related geometrically in that $\hat{S}$ is obtained from $S$ by the (-)transform and $S$ is obtained from $\hat{S}$ by the (+)transform. 
Thus, ruled minimal Lagrangian submanifolds of $\mathbb{C} P^{3}(4)$ induce two constructions on what could be a special class of minimal surfaces in $S^{5}(1)$, namely a (-)transform, given by (57), producing $\hat{S}$ from $S$ and a $(+)$ transform, given by (56), producing $S$ from $\hat{S}$.

In a forthcoming paper [4] we shall show that if we apply either of these constructions to an arbitrary minimal surface with non-circular non-degenerate ellipse of curvature in $S^{5}(1)$ then we obtain another minimal surface in $S^{5}(1)$. As a consequence of this we will show that every such minimal surface in $S^{5}(1)$ may be constructed locally in the manner described in the present paper from a ruled minimal Lagrangian submanifold of $\mathbb{C} P^{3}(4)$.

\section{REFERENCES}

[1] J. Bolton, C. Scharlach, L. Vrancken and L. M. Woodward, From certain minimal Lagrangian submanifolds of the 3-dimensional complex projective space to minimal surfaces in the 5-sphere, Proceedings of the Fifth Pacific Rim Geometry Conference, Tohoku University, Tohoku Math. Publ., 20(2001), pp. 23-31.

[2] J. Bolton, C. Scharlach and L. Vrancken, From surfaces in the 5-sphere to 3-manifolds in complex projective 3-space, Bull. Austral. Math. Soc., 66(2002), pp. 465-475.

[3] J. Bolton, F. Pedit and L.M. Woodward, Minimal surfaces and the affine Toda field model, J. reine angew. Math., 459(1995), pp. 119-150.

[4] J. Bolton and L. VRancken, Transforms of minimal surfaces of the 5-sphere, Preprint.

[5] R.L. Bryant, Second order families of special Lagrangian 3-folds, Preprint.

[6] I. Castro and L. Vrancken, Minimal Lagrangian submanifolds in $\mathbb{C} P^{3}$ and the sinh-Gordon equation. Dedicated to Shiing-Shen Chern on his 90th birthday, Results Math., 40:1-4 (2001), pp. 130-143.

[7] B.-Y. Chen, F. Dillen, L. Verstraelen and L. Vranchen, Totally real submanifolds of $\mathbb{C} P^{n}$ satisfying a basic equality, Arch. Math., 63(1994), pp. 553-564.

[8] B.-Y. Chen, F. Dillen, L. Verstraelen and L. Vrancken, An exotic totally real minimal immersion of $S^{3}$ in $\mathbb{C} P^{3}$ and its characterization, Proc. Royal Soc. Edinburgh, 126(1996), pp. 153-165.

[9] H. B. Lawson, Complete minimal surfaces in $S^{3}$, Ann. Math., 92(1970), pp. 335-374.

[10] H. Reckziegel, Horizontal lifts of isometric immersions into the bundle space of a pseudoRiemannian submersion, in Global Differential Geometry and Global Analysis (1984), Lecture Notes in Mathematics 1156, Springer Verlag (1985), pp. 264-279. 
J. BOLTON AND L. VRANCKEN 